\title{
Neutrophil apoptosis: impact of granulocyte macrophage colony stimulating factor on cell survival and viability in chronic kidney disease and hemodialysis patients
}

Nariman Zahran, Azza Sayed, Iman William, Ola Mahmoud, Omar Sabry, Manar Rafaat

Theodor Bilharz Research Institute, Imbaba Giza, Egypt

Submitted: 23 May 2011

Accepted: 19 October 2011

Arch Med Sci 2013; 9, 6: 984-989

DOI: 10.5114/aoms.2013.39789

Copyright @ 2013 Termedia \& Banach

\section{Abstract}

Introduction: Altered neutrophil apoptosis might be responsible for recurrent bacterial infections encountered in hemodialysis (HD) and chronic kidney disease (CKD) patients. This work was designed to assess the neutrophil apoptotic activity and the impact of implementation of granulocyte macrophage colony stimulating factor (GM-CSF), as a survival factor, on neutrophil apoptosis among these patients.

Material and methods: Twenty-five patients on regular HD along with 34 CKD patients on conservative treatment, as well as 15 healthy controls, were investigated for apoptotic rate via assessment of neutrophil expression of Annexin$\mathrm{V}$ by flow cytometry, before and after $20 \mathrm{~h}$ culture in absence and presence of GM-CSF. Neutrophil viability was determined using light microscopy. The preservation of neutrophil activation in these patients was analyzed by flow cytometric CD18 neutrophil expression. Chronic inflammatory state was evaluated by estimating C-reactive protein (CRP) and soluble intercellular adhesion molecule-1 (sICAM-1). Obtained data were statistically analyzed.

Results: Compared to controls, both HD and CKD groups had a significant increase of Annexin-V and CD18 expression and significant decrease in neutrophil viability. Culture of their neutrophils with GM-CSF showed significant decrease of apoptosis accompanied by improvement of neutrophil viability compared to their cultured cells without GM-CSF. These patients also showed significant elevation of CRP and SICAM-1.

Conclusions: Granulocyte macrophage colony stimulating factor demonstrated an evident impact on improving in vitro neutrophil survival and viability in HD and CKD patients. Therefore, this may represent promising preventive and/or therapeutic strategies against infection frequently observed in these patients and causing morbidity and mortality.

Key words: neutrophil apoptosis, annexin-V, granulocyte macrophage colony stimulating factor, chronic kidney disease, hemodialysis.

\section{Introduction}

Neutrophils are terminally differentiated cells that comprise the greatest cellular component of the immune system and play an important role in innate host defense against microbial infection [1]. A normal resolution of inflammation depends on the timely removal of cells from the site of inflammation, which is related to their ability to undergo apoptosis [2].

\author{
Corresponding author: \\ Dr. Ola Mahmoud MD \\ Theodor Bilharz \\ Research Institute \\ Imbaba Giza, Egypt \\ P.O. BOX: 30 Imbaba \\ Phone: +20224723116 \\ E-mail: olamahmoud5@ \\ hotmail.com
}


Although a number of agents have been shown to regulate apoptosis or necrosis in neutrophils, the mechanism deciding the final outcome still remains to be clarified [3]. Assuming that, phagocytosis of bacteria accelerates apoptosis significantly, subsequently facilitating clearance of effete neutrophils containing dead bacteria [4]. Conversely, some bacteria-derived products, such as lipopolysaccharides, prolong neutrophil survival [5]. Indeed, several proinflammatory cytokines, chemokines, or growth factors, including granulocyte macrophage colony stimulating factor (GM-CSF), delay neutrophil apoptosis, which seems likely important for effective clearance of invading microorganisms [6].

In vitro studies have shown that neutrophils from uremic patients undergo accelerated apoptosis; nevertheless, uremic plasma obtained from dialysis patients accelerates apoptosis in polymorphonuclear leukocytes (PMNs) from healthy donors [7]. During dialysis sessions, neutrophil activation and apoptosis acceleration are mainly triggered through antibody-dependent activation of complement components following contact of neutrophils with dialyzer membranes. Furthermore, the degree of spontaneous apoptosis of leucocytes is higher when bioincompatible membranes are used for hemodialysis (HD) than biocompatible ones [8].

The present work was undertaken to assess the neutrophil apoptosis in chronic kidney disease (CKD) and HD patients. Moreover, the extent of preserved neutrophil function among these patients was estimated. In addition, the influence of GMCSF on neutrophil apoptosis was evaluated in an attempt to clarify whether its use could have a potential therapeutic benefit in preventing or reducing severity of superimposed bacterial infections which may be fatal especially in those high risk patients with immunocompromised status.

\section{Material and methods}

\section{Subjects}

Fifty-nine patients were selected from the Nephrology Department and Dialysis unit of Theodor Bilharz Research Institute (TBRI), Egypt. The patients were divided into 25 with end stage renal disease (ESRD) (14 males and 11 females with mean age of $42.88 \pm 2.50)$ on regular hemodialysis; 3 sessions weekly, $4 \mathrm{~h}$ each, for a period of $\geq 3$ months using a Fresenius 4008 B machine, Hemophane filters with $1.4 \mathrm{~m}^{2}$ surface area and sodium acetate solution as a dialysate and 34 patients with CKD on conservative treatment (17 males and 17 females with mean age of $51.65 \pm 2.05$ ). In addition, 15 healthy subjects served as controls (10 males and 5 females with mean ages of $31.87 \pm 2.29$ ).

None of the patients had transfusion transmitted infection in the previous month, or chronic viral infections (e.g. hepatitis B, hepatitis C, human immunodeficiency virus), or was on any anti-inflammatory medication at the time of the study, or had previous transplants, or history of malignancy. Informed consent was obtained from all participants, and the protocol of the study was approved by the Ethics Institutional Review Committee of TBRI and was conducted in accordance with the Declaration of Helsinki (1975).

\section{Assay methods}

The following investigations were performed for all studied subjects. Reference ranges were provided by each manufacturer.

\section{Routine investigations}

All individuals enrolled in the study were subjected to automated haemogram (ACT Differential, Beckman, France), liver function tests (alanine aminotransferase and aspartate aminotransferase) and kidney function tests (creatinine and urea) (Hitachi 736, Hitachi, Japan). Hepatitis markers (HBs-Ag and HCV-Ab) and HIV were analyzed by means of enzyme linked immunoassay (ELISA) kits (Abbott Laboratories, North Chicago, IL). Determination of C-reactive protein (CRP) employed the RAVITEX® CPR Latex serology test (Omega Diagnostics, UK).

\section{Special investigations}

Neutrophil apoptosis activity and CD18 expression investigations were performed on neutrophils before and after culture in complete medium in a humidified atmosphere with $5 \% \mathrm{CO}_{2}$ at $37^{\circ} \mathrm{C}$ in absence and presence of recombinant human (rh)GM-CSF for $20 \mathrm{~h}$.

\section{Isolation of neutrophils}

Four $\mathrm{ml}$ of heparinized peripheral blood were collected from all subjects. The following steps were carried out under laminar flow and at $22^{\circ} \mathrm{C}$ to avoid blood contamination. Leucocyte rich plasma (LRP) was separated after $6 \%$ dextran sedimentation then fractionated using density gradient centrifugation with Percoll (Biochrom, AG) [9]. In brief, first, $2 \mathrm{ml}$ of Percoll stock solution (density 1.077) were added using a sterile Pasteur pipette slowly on the tube wall, then $2 \mathrm{ml}$ of Percoll (density 1.113) were added in the same way. Two $\mathrm{ml}$ of LRP were over-layered on the Percoll slowly and a few millimeters above the surface of the Percoll. The gradient was obtained by spinning in a swing-out rotor centrifuge (Eppendorf 5024) at $1600 \mathrm{~g}$ for $20 \mathrm{~min}$.

After centrifugation, neutrophils at the interface layer between Percoll and plasma were collected in a culture tube, then washed twice in phosphate 
buffer saline (PBS) at $1000 \mathrm{rpm}$ for $10 \mathrm{~min}$. The supernatant was discarded, and the cell pellet was resuspended in $1 \mathrm{ml}$ complete RPMI 1640 medium with stable glutamine (EuroClone, IBS, France).

\section{Neutrophil viability by trypan blue}

One volume of $0.4 \%$ trypan blue was added to 5 volumes of the previously prepared cell suspension in a sterile microcentrifuge tube and incubated at room temperature for 5 min, then counted in a hemocytometer [10]. In all cases, more than $98 \%$ of PMNs were viable by trypan blue dye exclusion.

\section{Neutrophil culture}

Neutrophils were resuspended at a final concentration of $2 \times 10^{6} / \mathrm{ml}$ in complete RPMI 1640 medium supplemented with $10 \%$ heat inactivated fetal bovine serum (EuroClone, IBS. France) and $1 \mathrm{ml}$ of penicillin streptomycin solution 100× (EuroClone IBS. France) and mixed well. Neutrophils were incubated, for $20-22 \mathrm{~h}$ at $37^{\circ} \mathrm{C}$ in $5 \% \mathrm{CO}_{2}$ in absence and presence of $100 \mathrm{ng} / \mathrm{ml} \mathrm{rh}-\mathrm{GM}-\mathrm{CSF}$ (R\&D systems) [11].

\section{Measurement of neutrophil apoptosis by flow cytometry}

The percentage of apoptotic neutrophils was assessed by Annexin V-FITC/propidium iodide (PI) binding kit, (BD Pharmagen ${ }^{\mathrm{TM}}$ ) using a flow cytometry $\left(C\right.$ OULTER ${ }^{\circledR}$ EPICS $^{\circledR}$ XL $^{\text {TM }}$, USA) before and after culture with and without addition of rh-GM-CSF according to the manufacturer's specifications [12].

Table I. Results of studied parameters in different groups

\begin{tabular}{|c|c|c|c|}
\hline Parameter & $\begin{array}{l}\text { Control } \\
(n=15)\end{array}$ & $\begin{array}{c}\mathrm{HD} \\
(n=23)\end{array}$ & $\begin{array}{c}\text { CKD } \\
(n=33)\end{array}$ \\
\hline $\begin{array}{l}\text { Urea } \\
{[\mathrm{mg} / \mathrm{dl}]}\end{array}$ & $21.07 \pm 1.21$ & $126.76 \pm 9.40^{a а}$ & $116.97 \pm 7.96^{\text {aa }}$ \\
\hline $\begin{array}{l}\text { Creatinine } \\
{[\mathrm{mg} / \mathrm{dl}]}\end{array}$ & $0.76 \pm 0.05$ & $5.98 \pm 0.40^{a a}$ & $4.25 \pm 0.42^{a a b}$ \\
\hline CRP $[\mathrm{mg} / \mathrm{l}]$ & $6.07 \pm 0.07$ & $59.00 \pm 16.34^{a}$ & $78.69 \pm 12.99$ aа \\
\hline $\begin{array}{l}\text { sICAM-1 } \\
{[\mathrm{ng} / \mathrm{ml}]}\end{array}$ & $6.53 \pm 0.55$ & $24.53 \pm 1.54^{a}$ & $20.14 \pm 1.06^{a b b}$ \\
\hline $\begin{array}{l}\text { WBCs } \\
{\left[\times 10^{3} / \mu l\right]}\end{array}$ & $6.47 \pm 0.44$ & $7.98 \pm 0.69$ & $9.00 \pm 0.74^{a}$ \\
\hline $\begin{array}{l}\text { Neutrophils } \\
{\left[\times 10^{3} / \mu \mathrm{l}\right]}\end{array}$ & $3.45 \pm 2.27$ & $5.19 \pm 2.39$ aа & $6.09 \pm 1.50$ aа \\
\hline $\begin{array}{l}\text { Annexin V } \\
{[\%]}\end{array}$ & $5.17 \pm 0.66$ & $9.55 \pm 0.99^{a}$ & $15.68 \pm 1.09$ aabb \\
\hline $\begin{array}{l}\text { Viability } \\
\text { [\%] }\end{array}$ & $94.81 \pm 0.68$ & $90.31 \pm 1.00^{a}$ & $84.22 \pm 1.07$ aabb \\
\hline CD18 [\%] & $8.87 \pm 1.05$ & $48.41 \pm 3.95^{\mathrm{aa}}$ & $43.71 \pm 2.64 \mathrm{aa}$ \\
\hline
\end{tabular}

Values are expressed as mean $\pm S E ; a_{p}<0.05$ and $a a_{p}<0.01$ relative to control group; $b_{p}<0.01$ and $b b_{p}<0.01$ relative to HD group
Cells that stained only for Annexin-V were considered in early apoptosis, and those that dually stained for Annexin- $\mathrm{V}$ and $\mathrm{PI}$ were indicated as being in late apoptosis or necrosis. Cells negative for Annexin-V/PI were considered to be viable.

\section{Determination of the surface expression of CD18}

Using flow cytometry, immunostaining with R-phycoerythrin-conjugated antibodies (IOTest ${ }^{\circledR}$ CD18-PE), which were purchased from Immunotech, Beckman Coulter, Inc., together with specific isotypic control reagent, was performed. The density of CD18 on neutrophils was presented as mean fluorescence intensity of the gated leucocyte population within a chosen field.

\section{Assessment of apoptosis by light microscopy}

Cytospin preparations were fixed in methanol, stained with Leishman-Giemsa and examined at oil magnification. Apoptotic PMNLs were identified using morphological criteria of apoptosis (chromatin condensation, fragmented nuclei, cell shrinkage and the presence of cytoplasmic vacuolization). For all samples 200-400 cells per slide were examined and the number of apoptotic cells was expressed as the percentage of the total cells on the slide [13].

\section{Determination of soluble intercellular adhesion molecule-1}

Serum intercellular adhesion molecule-1 (ICAM-1) levels were assayed by sandwich ELISA technique (R\&D Systems, Inc. Minneapolis, USA) according to the manufacturer's instructions. Each sample was assayed in duplicate.

\section{Statistical analysis}

All data were represented by means \pm standard error of mean (SE). SPSS computer program (version 12 windows) was used. Comparison between mean values of pre- and post-incubation time with in the same group was performed using paired $t$ test, while between different groups one-way analysis of variance (ANOVA) with post hoc test using least significant difference was used. Correlation between parameters using Spearman's rank correlation coefficient, in which $p$ value $<0.05$, was considered significant, and $p<0.01$ highly significant [14].

\section{Results}

Routine investigations indicated highly significant increase of serum urea and creatinine levels $(p<0.01)$ for both HD and CKD groups compared to control subjects (Table I). 
As regards the results of microinflammatory state parameters, HD and CKD patients showed statistically significant increases in absolute neutrophil count $(p<0.01)$, CRP $(p<0.05 ; p<0.01$ respectively) and SICAM $(p<0.05)$ compared to controls. Moreover, a statistically significant increase in sICAM-1 $(p<0.01)$ was also observed when comparing HD patients with CKD patients (Table I).

Hemodialysis and CKD patients showed a significant increase in neutrophil surface expression of Annexin- $\mathrm{V}$ ( $p<0.05 ; p<0.01$ respectively) denoting accelerated apoptosis and a significant decrease in percentage of neutrophil viability $(p<0.01)$ compared to controls. Moreover, neutrophils from CKD patients exhibited significantly higher Annexin-V expression and lower viability $(p<0.01)$ compared to HD patients. In addition, there was a significant increase of neutrophil activation among HD and CKD patients in comparison to healthy subjects, proved by CD 18 expression $(p<0.01)$ (Table I).

Cultured neutrophils without addition of GM-CSF showed a significant increase in neutrophil apoptosis and decrease in neutrophil viability $(p<0.05)$ in HD compared to baseline levels before culture. Meanwhile, a significant inhibition of neutrophil apoptosis $(p<0.01)$ and increase in neutrophil viability $(p<0.05)$ was observed in neutrophils cultured with GM-CSF in both CKD and HD groups (Table II).

A correlation analysis between Annexin- $V$ expression and the other studied parameters was demonstrated in (Table III).

\section{Discussion}

Uremia-related immune dysfunction is a complex interaction between the innate and adaptive systems, in which persistent immune activation and immune suppression coexist [15]. The pathogenic mechanisms of these immunological abnormalities have been ascribed in part to uremic toxins, malnutrition, iron overload, dialysis-related factors, and possibly apoptosis [16-19].
Chronic low-grade inflammation is a common feature of CKD and HD patients due to multiple factors including uremia, volume overload, co-morbidity and the dialysis procedure per se [20, 21]. C-reactive protein and SICAM are said to independently predict mortality in CKD patients [22]. In the present work, a significant increase in the inflammatory markers CRP and SICAM-1 was observed in both HD and CKD groups compared to controls. Moreover, the HD group revealed a significant increase in the sICAM-1 compared to the CKD group. Elevated CRP and SICAM-1 was referred to exposure to inflammatory stimuli which might be aggravated by superimposed infection [20, 23]. In the same context, total white blood count (WBC) and absolute neutrophil counts are considered to be neutrophil-derived inflammation markers [24]. In the current study, although the mean values did not exceed the upper normal range, the absolute neutrophil counts showed a highly significant increase in both HD and CKD groups when com-

Table II. Comparison of annexin V and neutrophil viability measured before and after culture with and without GM-CSF in patient groups

\begin{tabular}{|lcc|}
\hline Variable & $\begin{array}{c}\text { CKD group } \\
(n=33)\end{array}$ & $\begin{array}{c}\text { HD group } \\
(n=23)\end{array}$ \\
\hline $\begin{array}{l}\text { Before culture (Day 1) } \\
\text { Annexin V }\end{array}$ & $15.68 \pm 1.09$ & $9.55 \pm 0.99$ \\
\hline Neutrophil availability & $84.22 \pm 1.07$ & $90.31 \pm 1.00$ \\
\hline \begin{tabular}{l} 
After culture without GM-CSF $($ Day 2$)$ \\
\hline Annexin V
\end{tabular} & $18.32 \pm 1.86$ & $16.39 \pm 2.81^{\mathrm{a}}$ \\
\hline $\begin{array}{l}\text { Neutrophil availability } \\
\text { After culture with GM-CSF (Day } 2)\end{array}$ & $83.56 \pm 1.7$ & $81.18 \pm 3.42^{\mathrm{a}}$ \\
\hline Annexin V & $13.17 \pm 1.65^{\mathrm{bb}}$ & $11.32 \pm 1.98^{\mathrm{bb}}$ \\
\hline Neutrophil availability & $87.18 \pm 1.66^{\mathrm{b}}$ & $86.30 \pm 3.20^{\mathrm{b}}$ \\
\hline
\end{tabular}

Values are expressed as mean $\pm S E ; a_{p}<0.05$ significant difference relative to baseline value before culture; $b_{p}<0.05$ and $b b_{p}<0.01$ significant difference relative to neutrophil incubated without GM-CSF

Table III. Correlation between the percentage of annexin $\vee$ and different studied parameters in the two studied groups

\begin{tabular}{|lcccc|}
\hline Correlated variable & \multicolumn{2}{c|}{ HD group $(n=25)$} & \multicolumn{2}{c|}{ CKD group $(n=34)$} \\
\cline { 2 - 5 } & $r$ & $p$ & 0.071 & $p$ \\
\hline Neutrophil count & 0.666 & $0.0001^{* *}$ & 0.579 & $0.689^{\mathrm{NS}}$ \\
\hline CD18 & -0.403 & $0.046^{\star}$ & -0.025 & $0.0001^{\star *}$ \\
\hline sICAM-1 & -0.153 & $0.465^{\mathrm{NS}}$ & 0.137 & $0.898^{\mathrm{NS}}$ \\
\hline CRP & 0.491 & $0.039^{*}$ & -0.199 & $0.505^{\mathrm{NS}}$ \\
\hline Creatinine & -0.045 & $0.832^{\mathrm{NS}}$ & -0.158 & $0.301^{\mathrm{NS}}$ \\
\hline Urea & 0.071 & $0.734^{\mathrm{NS}}$ & 0.124 & $0.414^{\mathrm{NS}}$ \\
\hline WBCs & 0.421 & $0.036^{\star}$ & -0.993 & $0.486^{\mathrm{NS}}$ \\
\hline Viable & -0.999 & $0.001^{\star *}$ & $0.01^{* *}$ \\
\hline
\end{tabular}

NS - not significant $(p>0.05),{ }^{*} p<0.05$ - significant, ${ }^{* *} p<0.01-$ highly significant, $r$ - correlation coefficient 
pared to the control group. Lack of neutrophilia in spite of presence of a microinflammatory state could be attributed to accelerated apoptosis in these patients as recorded in the present study.

In the present study freshly harvested neutrophils demonstrated significant acceleration in the apoptotic rates among both CKD and HD groups compared to controls. In addition, the CKD group showed a significant increase in neutrophil apoptosis compared to the HD group. Consequently, the dialysis process may slow the observed acceleration of neutrophil apoptosis in these patients. Indeed, most inflammation-related processes alter neutrophil apoptosis which consequently becomes dysfunctional. This dysfunctional pattern is similar to that of uremic neutrophils, which demonstrate altered oxidative burst, impaired chemotaxis, aggregation and phagocytosis. Therefore, it is possible to speculate that the existence of a mixture of a large number of various toxic uremic solutes in serum of CKD patients may accelerate neutrophil apoptosis [25]. Moreover, several studies have revealed that dialysis may correct or normalize apoptosis rates through clearance of serum toxic uremic solutes in ESRD patients [26-28]. The accelerated neutrophil apoptotic process in vivo may limit the release of neutrophil contents from disintegrating cells and prevent further injuries to epithelial cells induced by neutrophils [29]. On the other hand, because apoptotic neutrophils are functionally impaired [7], it seems reasonable to speculate that uremiainduced apoptosis may be partly responsible for the risk of secondary infection commonly encountered in patients with CKD [25].

The present results of freshly harvested neutrophils also revealed a significant increase in neutrophil surface expression of CD18 in CKD and HD patients compared to healthy individuals, whereas no significant difference was noticed between CKD and HD groups. These results are in accordance with other studies and reflect the state of neutrophil activation found in these patients mainly due to uremic toxin solutes and/or bacterial infections [30]. Increased neutrophil activation, up-regulation of CD18 and neutrophil adhesion through CD18 may be in part responsible for enhanced neutrophil apoptosis in uremic patients [31, 32]. This finding was clarified by the significant direct correlation between neutrophil apoptosis (Annexin-V) and neutrophil surface expression of CD18 in CKD and HD patients studied.

Results of untreated neutrophils cultured for $20 \mathrm{~h}$ revealed a higher percentage of apoptotic rates and decrease in cell viability in absence of GM-CSF compared to freshly harvested neutrophils, which was significant in the HD group. In accordance, a study conducted on healthy individuals demonstrated that whole blood samples cultured for $20 \mathrm{~h}$ contained a markedly higher percentage of apoptotic neutrophils compared to before culture. This post-culture increased apoptotic rate was interpreted by the fact that preserved cytokines and other factors naturally present in plasma could modulate apoptosis [7]. Adding GM-CSF to the cultured neutrophil significantly delayed neutrophil apoptosis and increased neutrophil viability in both CKD and HD groups compared to corresponding cultured cells without GM-CSF. Similar results were observed in neutrophils from healthy individuals $[29,33,34]$ and they suggested that the exact mechanisms that mediate the pro-survival effects of GM-CSF seem to happen through the mitochondrial pathway by preventing Bax translocation, cytochrome c release and subsequent caspase 3 activation.

It could be concluded that the survival factor GM-CSF has an important impact in reducing neutrophil apoptosis and improving cell survival in HD and CKD patients. So, depending on the mentioned results, and the known promoting effect of GM-CSF on neutrophil functions, the therapeutic use of this factor may represent a useful new strategy for treatment of infections, which seem to occur more frequently in the setting of kidney disease and are associated with poor outcomes compared with the general population. Eventually, additional in vivo studies are needed, to elucidate the impact of GMCSF on decreasing neutrophil apoptosis and increasing resistance to infections, at the clinically meaningful level.

\section{References}

1. Faurschou M, Borregaard N. Neutrophil granules and secretory vesicles in inflammation. Microbes Infect 2003; 5: 1317-27.

2. Haslett C, Savill JS, Whyte MKB, Stern M, Dransfield I, Meagher LC. Granulocyte apoptosis and the control of inflammation. Philos Trans R Soc Lond B 1994; 345: 327-33.

3. Arash SS, Heather A, Abdelilah SG. The molecular mechanisms of glucocorticoids-mediated neutrophil survival. Current Drug Targets 2011; 12: 556-62.

4. Kobayashi SD, Braughton KR, Whitney AR, et al. Bacterial pathogens modulate an apoptosis differentiation program in human neutrophils. Proc Natl Acad Sci USA 2003; 100: 10948-53.

5. Hachiya O, Takeda Y, Miyata H, Watanabe H, Yamashita T, Sendo F. Inhibition by bacterial lipopolysaccharide of spontaneous and TNF-alpha- induced human neutrophil apoptosis in vitro. Microbiol Immunol 1995; 39: 715-23.

6. Kobayashi SD, Voyich JM, Whitney AR, DeLeo FR. Spontaneous neutrophil apoptosis and regulation of cell survival by granulocyte macrophage-colony stimulating factor. J Leuk Biol 2005; 78: 1408-18.

7. Majewska E, Baj Z, Sulowska Z, Rysz J, Luciak M. Effects of uraemia and haemodialysis on neutrophil apoptosis and expression of apoptosis-related proteins. Nephrol Dial Transplant 2003; 18: 2582-8.

8. Rosenkranz AR, Peherstorfer E, Körmöczi GF et al. Complement-dependent acceleration of apoptosis in 
neutrophils by dialyser membranes. Kidney Int 2001; 59 S216-220.

9. Freitasa M, Portob G, Limaa JLFC, Fernandes E. Isolation and activation of human neutrophils in vitro. The importance of the anticoagulant used during blood collection. Clin Biochem 2008; 41: 570-5.

10. Aref S, El Refaei M, Goda T, Sakrana M, El-Nemre H. Accelerated neutrophil apoptosis in neutropenic patients with hepatosplenic schistosomiasis is induced by serum Fas ligand. Hematol J 2004; 5: 434-9.

11. Derouet M, Thomas L, Cross A, Moots RJ, Edwards SW. Granulocyte macrophage colony stimulating factor signaling and proteasome inhibition delay neutrophi apoptosis by increasing the stability of Mcl-1. J Biol Chem 2004; 279: 26915-21.

12. Hu Z, Sayeed MM. Suppression of mitochondria-dependent neutrophil apoptosis with thermal injury. Am J Physiol Cell Physiol 2004; 286: C170-8.

13. Doonan F, Cotter TG. Morphological assessment of apoptosis. Methods 2008; 44: 200-4.

14. Petei A, Sabin C. Medical statistics at Glance. Blackwell Science, London 2001

15. Hauser AB, Stinghen AEM, Kato $S$, et al. Characteristics and causes of immune dysfunction related to uremia and dialysis. Perit Dial Int 2008; 28: S183.

16. De Smet R, Vanholder R. Pathophysiologic effects of uremic retention solutes. J Am Soc Nephrol 1999; 10: 18215-32.

17. Hörl WH. Neutrophil function and infections in uremia. Am J Kidney Dis 1999; 33: xlv-xlviii.

18. Himmelfarb J, Hakim RM. Biocompatibility and risk of infection in hemodialysis patients. Nephrol Dial Transplant 1994; 9 Suppl 2: 138-44.

19. Cohen G, Rudnicki M, Hörl WH. Uremic toxins modulate the spontaneous apoptotic cell death and essential functions of neutrophils. Kidney Int Suppl 2001; 78: S48-52.

20. Gad MZ, El-Mesallamy HO, Sanad EF. HsCRP, sICAM-1 and TAFI in hemodialysis patients: linking inflammation and hypofibrinolysis to cardiovascular events. Kidney Blood Press Res 2008; 31: 391-7.

21. Perianayagam M, Balakrishnan V, Guo D, Pereira B, Jaber B. Quantification of $\mathrm{Bax}$ and $\mathrm{BCl} 2$ in polymorphonuclear leukocytes from haemodialysis patients: relation to hydrogen peroxide. Euro J Clin Invest 2003; 33: 905-11.

22. Stenvinkel P. C-reactive protein: does it promote vascular disease? Nephrol Dial Transplant 2006; 21: 2718-20.

23. Vaccaro F, Mule G, Cottone $S$, et al Circulating levels of adhesion molecules in chronic kidney disease correlate with the stage of renal disease and with C-reactive protein. Arch Med Res 2007; 38: 534-8.

24. Sela S, Swirski R, Mazor R, et al. Primed peripheral polymorphonuclear leucocytes: a culprit underlying chronic low-grade inflammation and systemic oxidative stress in chronic kidney disease. J Am Soc Nephrol 2005; 16: 2431-8.

25. Cohen G, Raupachova J, Wimmer T, Deicher R, Horl W. The uremic retention solute para-hydroxy-hippuric acid attenuates apoptosis of polymor-phonuclear leukocytes from healthy subjects but not from hemodialysis patients. Nephrol Dial Transplant 2008; 23: 2512-9.

26. Sardenberg C, Suassuna P, Claudia M, Andreoli C. Effects of uremia and dialysis modality on polymorphonuclear cell apoptosis and function. Nephrol Dial Transplant 2006; 21: 160-5.

27. Kennedy A, DeLeo F. Neutrophil apoptosis and the resolution of infection. Immunol Res 2009; 43: 8049-56.

28. Cendoroglo M, Jaber BL, Balakrishnan VS, Perianayagam M, King AJ, Pereira BJG. Neutrophil apoptosis and dysfunction in uremia. JASN 1999; 1: 93-100.
29. Kobayashi S, Voyich M, Whitney A, DeLeo FR. Spontaneous neutrophil apoptosis and regulation of cell survival by granulocyte macrophage colony stimulating factor. J Leuko Biol 2005; 78: 1408-18.

30. Caimi G, Montana M, Carollo C. Polymorphonuclear leukocyte integrin in chronic renal failure. Clin Hemorheol Microcirc 2005; 32: 43-9.

31. Wang SZ, Smith PK, Lovejoy M, Bowden JJ, Alpers JH, Forsyth KD. The apoptosis of neutrophils is accelerated in respiratory syncytial virus (RSV)-induced bronchiolitis. Clin Exp Immunol 1998; 114: 49-54.

32. Sim S, Park SJ, Yong TS, Shin MH. Involvement of beta 2-integrin in ROS-mediated neutrophil apoptosis induced by Entamoeba histolytica. Microbes Infect 2007; 9: 1368-75.

33. Molloy EJ, O'Neill AJ, Grantham JJ, Sheridan-Pereira M, Fitzpatrick JM, Webb DW, Watson RW. Granulocyte colonystimulating factor and granulocyte-macrophage colonystimulating factor have differential effects on neonatal and adult neutrophil survival and function. Pediatr Res 2005; 57: 806-12.

34. Wolach B, van der Laan LJ, Maianski NA, et al. Growth factors G-CSF and GM-CSF differentially preserve chemotaxis of neutrophils aging in vitro. Exp Hematol 2007; 35: 541-50. 